\title{
Atividade anti-retroviral e propriedades farmacocinéticas da associação entre lamivudina e zidovudina
}

\author{
Jacqueline de Souza $^{1 *}$, Sílvia Storpirtis ${ }^{2}$
}

${ }^{1}$ Departamento de Farmácia, Escola de Farmácia, Universidade Federal de Ouro Preto, ${ }^{2}$ Departamento de Farmácia, Faculdade de Ciências Farmacêuticas, Universidade de São Paulo.

*Correspondência:

J. de Souza

Escola de Farmácia - UFOP

Rua Costa Sena, 171 - Centro

35400-000 Ouro Preto - MG - Brasil

E-mail: jacquelibebh@bol.com.br
A lamivudina (3TC) e a zidovudina (ZDV) são agentes antiretrovirais indicados para o tratamento de infecção pelo HIV. Eles são análogos nucleosídeos, que passam por reações intracelulares de fosforilação e atuam inibindo a enzima transcriptase reversa. Os compostos trifosforilados formados se ligam à cadeia de DNA pró-viral impedindo sua replicação. A terapêutica anti-retroviral utilizando apenas um fármaco ocasiona, freqüentemente, a seleção de cepas resistentes. Os efeitos adversos provocados pela $Z D V$ são mais comuns em pacientes com doença em estágio avançado, sendo o principal deles a mielossupressão. A $3 T C$ é bem tolerada, mas sua toxicidade aumenta proporcionalmente ao acréscimo da dose. Quanto à farmacocinética, ambos são facilmente absorvidos passivamente e biotransformados em compostos ativos trifosforilados. Eles são amplamente distribuidos, penetram livremente nos tecidos a partir da circulação sistêmica. Difundemse através da placenta da circulação materna para a circulação fetal. O principal caminho metabólico da ZDV é a glicuronidação, cerca de 60 a 70\% do fármaco é inativado por essa via. Ela é eliminada rapidamente, sendo sua meia-vida de eliminação da ordem de 1 a 1,5 h. Apenas 5\% a 10\% da 3TC são biotransformados em um metabólito trans-sulfóxido inativo. Sua meia-vida de eliminação é de 8,5 a 11,5 h, sendo que 70\% do fármaco são excretados de forma inalterada na urina até 24 h após a dose.
Unitermos

- Lamivudina

- Zidovudina

- Anti-retrovirais

- Farmacocinética

\section{INTRODUÇÃO}

A síndrome de imunodeficiência adquirida (AIDS) é uma doença caracterizada por significativa imunossupressão associada a infecções oportunistas, neoplasias secundárias e manifestações neurológicas (Neto et al., 1996).
A AIDS foi detectada inicialmente, em 1981. Os estudos foram iniciados após surgimento de vários casos de sarcoma de Kaposi e pneumonia pelo Pneumocistis carinii, em pacientes homossexuais masculinos. Estes eram procedentes de grandes cidades norte-americanas (Barry et al., 1999; Balint, 2001). Embora essas fossem 
doenças já conhecidas, elas haviam sido detectadas anteriormente em indivíduos idosos e pacientes com câncer em estágios avançados, respectivamente. A incidência em homossexuais masculinos chamou a atenção do Centro para o Controle e Prevenção de Doenças (CDC - "Center for Disease Control and Prevention"). O CDC, órgão norte-americano de vigilância epidemiológica, passou a estudar a doença e definir seu perfil clínicos e epidemiológico. A descoberta do agente causador da AIDS só ocorreu em 1984, sendo posteriormente classificado como um retrovírus e denominado Vírus da Imunodeficiência Humana (HIV) (Amato Neto et al., 1996). Atualmente são conhecidos os vírus HIV tipos 1 e 2. Devido às características epidemiológicas da AIDS estudos sugerem uma etiologia infecciosa, transmitida por via sexual, vertical e parenteral (Amato Neto et al., 1996).

No Brasil, os primeiros casos de AIDS surgiram em 1982 e, atualmente, existem cerca de 130.000 pessoas doentes no País. Calcula-se que para cada pessoa que apresente os sintomas existam cerca de 5 a 50 pessoas assintomáticas e que desconhecem que estão infectadas (Brasil, 2002a).

Atualmente, o Ministério da Saúde adotou política de controle da disseminação da AIDS no País e assistência aos indivíduos infectados pelo HIV. Esta inclui, entre várias outras iniciativas, um programa de acesso universal e gratuito aos medicamentos anti-retrovirais na rede pública de saúde (Brasil, 2002b).

$\mathrm{Na} 11^{\circ}$ Conferência Internacional sobre a AIDS, que ocorreu em Vancouver, foi apresentada uma teoria para erradicação da doença pelo tratamento agressivo usando a combinação de agentes anti-retrovirais (Cohn, 1997).

Para inibir a replicação viral três classes de fármacos foram desenvolvidas: os inibidores da protease; os inibidores da transcriptase reversa nucleosídeos e os nãonucleosídeos (Aymard, 2000).

Em março de 1987, foi aprovado para comercialização, pelo órgão norte-americano de controle sobre produtos farmacêuticos FDA (Food and Drug Administration), o primeiro fármaco com atividade antiretroviral: a azidotimidina ou zidovudina (AZT ou ZDV), que foi utilizada para o tratamento de portadores do HIV (Styrt et al., 1996). Ele inibe o vírus HIV-1, HIV-2, o vírus-1 da leucemia T/linfoma em humanos e outros retrovírus de mamíferos (Aoki, 1999). Em 1995, a lamivudina (3TC) teve seu uso aprovado pelo FDA (Styrt et al., 1996). Ela atua inibindo a replicação do vírus HIV e alguns trabalhos relatam, ainda, sua atividade contra o vírus da hepatite B (Zhou, Sommadossi, 1997).

Ambos os fármacos são agentes anti-retrovirais da classe dos inibidores da transcriptase reversa nucleosí- dicos. Atualmente, eles são utilizados na terapia antiretroviral combinada com o objetivo de aumentar a supressão viral, prevenir a resistência aos fármacos e simplificar a posologia (Havlir, Lange, 1998).

A administração conjunta de ZDV e 3TC e mais um inibidor da protease ocasionou aumento de $3,5 \%$ no sucesso da terapêutica, comparativamente ao uso dos mesmos fármacos em comprimidos separados (Eron et al., 2000). A complexidade da terapêutica anti-retroviral, devido à variedade dos regimes de dosagem, associados a toxicidade, muitas vezes contribuem para erros ou não aderência ao tratamento pelos pacientes o que pode ser minimizado pelo uso de um medicamento contendo associação de ZDV e 3TC (Moyer et al., 1999).

\section{CARACTERÍSTICAS FÍSICO-QUÍMICAS}

A ZDV é semelhante à timidina endógena, diferenciando apenas pela presença de um grupo azido $\left(\mathrm{N}_{3}\right)$ no anel ribose (Veal, Back, 1995; Aoki, 1999) ao invés de hidroxila na posição 3. Ela apresenta-se neutra na faixa de $\mathrm{pH}$, que varia de 3,5 a 5,5 e pKa igual a 9,8 (Mesplet et al., 2001)

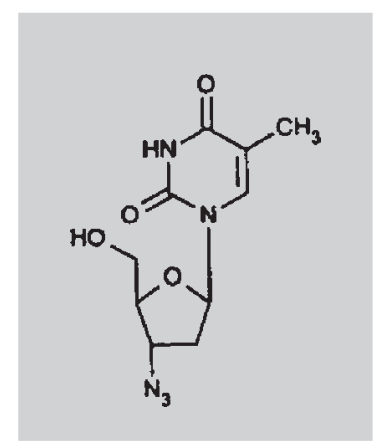

FIGURA 1 - Fórmula estrutural da zidovudina (ZDV) (Veal, Back, 1995).

A 3 TC é uma citidina sintética, 2', 3'didesoxinucleosídeo, com configuração absoluta $2 R, 5 S$ não natural (Perry, Faulds, 1997). Ela é uma didesoxipirimidina, em que o carbono 3' do anel da ribose é substituído por enxofre. A 3 TC é o enantiômero (-); o enantiômero $(+)$ possui maior toxicidade (Johnson et al., 1999).

A 3TC apresenta-se como pó branco a branco-amarelado, com pKa igual a 4,3 (protonação do grupo $\mathrm{NH}_{2}$ ). Este fármaco, quando dissolvido em água destilada, encontra-se primariamente sob a forma não-ionizada. $\mathrm{O} \mathrm{pH}$ da solução aquosa a $1 \%(\mathrm{p} / \mathrm{V})$ é 6,9 . Dados da literatura afirmam que tanto em estado sólido quanto em solução 


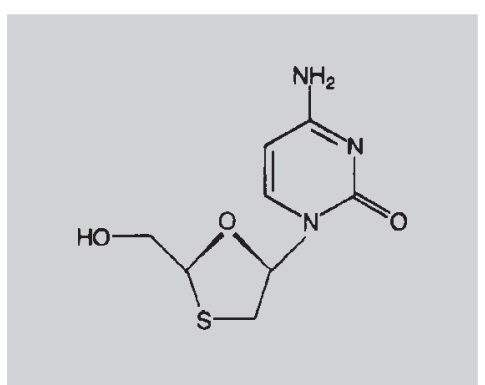

FIGURA 2- Fórmula estrutural da lamivudina (3TC) (Johnson et al., 1999).

aquosa ele possui elevada estabilidade à luz e à temperatura (Jozwiakowski et al., 1996; Kyoko et al., 1997; Fernandes, 2001).

\section{INDICAÇÕES TERAPÊUTICAS E DOSE}

A ZDV é indicada para o tratamento inicial de adultos infectados pelo HIV, apresentando contagem de células CD4+ inferior a 500 células $/ \mathrm{mL}$. Seu uso é indicado também para crianças com mais de três meses de idade e mulheres grávidas infectadas pelo HIV e seus recém-nascidos (Aoki, 1999).

A terapêutica anti-retroviral utilizando ZDV isoladamente freqüentemente ocasiona a seleção de cepas resistentes. Esquemas posológicos combinados têm se mostrado mais eficazes na supressão da replicação viral. Isto é comprovado por estudos comparando vários tratamentos associando os seguintes inibidores da transcriptase reversa: nevirapina, didanosina, zalcitabina e ZDV. Estes estudos demonstraram que o uso da terapia tripla contendo anti-retrovirais proporcionou decréscimo plasmático de RNA do HIV-1 a níveis abaixo do limite de detecção (Montaner et al., 1998; Henry et al., 1998).

A 3TC é usada no tratamento de infecção por HIV1 em combinação com outros agentes anti-retrovirais (Johnson et al., 1999). Estudos relatam sua atividade contra cepas de HIV resistentes à ZDV (Zhou, Sommadossi, 1997). Esquemas terapêuticos empregando a 3 TC em dose de $150 \mathrm{mg}$ duas vezes ao dia são indicados para adultos e adolescentes com mais de $50 \mathrm{mg}$. Em adultos com menos de $50 \mathrm{~kg}$ a dose deve ser ajustada para $2 \mathrm{mg} / \mathrm{kg}$ de peso duas vezes ao dia. Crianças com idade entre três meses e um ano tem como indicação de dose $4 \mathrm{mg} / \mathrm{kg}$, duas vezes ao dia (Perry, Faulds, 1997).

A 3TC é utilizada também no tratamento de pacientes com infecção crônica por HBV. Administrada em monoterapia com dose de $100 \mathrm{mg}$ por dia, ela é capaz de reduzir os níveis de HBV-DNA e normalizar a taxa de aminotransferase.
Um estudo duplo cego realizado em 97 pacientes portadores do HIV avaliou a eficácia de três condições terapêuticas: a utilização de $800 \mathrm{mg}$ de indinavir a cada oito horas; o uso conjunto de $200 \mathrm{mg}$ de ZDV três vezes ao dia mais $150 \mathrm{mg}$ de 3TC duas vezes ao dia ou a associação dos três medicamentos. Os pesquisadores observaram pacientes que usaram pela primeira vez a terapia composta pelos três anti-retrovirais conjuntamente e concluíram que a contagem de RNA-HIV se reduziu a menos de 500 cópias $/ \mathrm{mL}$ por mais de um ano. Isto foi atribuído à potente atividade anti-retroviral do indinavir e ao efeito sustentável da combinação de ZDV e 3TC (Gulick et al., 1997).

A terapia anti-retroviral combinada pode aumentar a supressão viral, prevenir a resistência aos fármacos e simplificar a posologia. Porém, alguns antagonismos farmacológicos podem ser observados, ocasionando concentrações plasmáticas sub-terapêuticas ou toxicidade inesperada. É observada profunda e sustentável supressão da doença ocasionada pelo HIV, quando são associados os seguintes anti-retrovirais: indinavir, ZDV e 3TC (Havlir, Lange, 1998).

Estudos realizados por Horneff e colaboradores (1998) avaliaram a tolerância e a eficácia da associação de ZDV e $3 \mathrm{TC}$ em crianças infectadas pelo HIV. Elas não haviam sido expostas a tratamento anteriormente. $\mathrm{O}$ estudo foi realizado em treze crianças de 4 meses a 10 anos. Dentre os pacientes apenas um teve que descontinuar o tratamento devido ao desenvolvimento de neutropenia e observação de que após 6 meses não havia significativa redução da carga viral. A terapia combinada de ZDV/3TC é promissora pois vírus resistentes a ZDV são fenotipicamente sensíveis à lamivudina pela mutação do resíduo 184. Além disso, é pequena ou inexistente a coresistência entre ambos (Horneff et al., 1998). Recentes estudos avaliaram as propriedades farmacocinéticas e farmacodinâmicas dos agentes anti-retrovirais. Foram avaliados modelos matemáticos visando correlacionar a dose terapêutica e o efeito em diminuir a carga viral. Embora estes modelos não tenham sido totalmente adequados para a quantificação do efeito máximo dos fármacos, eles possibilitam prever o sinergismo entre eles, bem como fornecem indícios sobre a resistência viral (Legrand et al., 2003)

\section{MECANISMO DE AÇÃO}

A ZDV é um pró-fármaco, cuja forma ativa é a zidovudina trifosfato (ZDV-3P). A ZDV-3P é obtida por reações de fosforilação do fármaco nos linfócitos. Seu mecanismo de ação consiste em inibir competitivamente 
a incorporação, pela enzima transcriptase reversa, da timidina ao DNA viral. (Veal, Back, 1995).

Para atuar, a 3TC também passa por reações intracelulares de fosforilação. A desoxicitidina quinase é responsável pela formação da lamivudina monofosfato (3TC-P). A lamivudina difosfato (3TC-2P) é formada pela ação das enzimas citidina monofosfato quinase e desoxicitidina monofosfato quinase. Finalmente, pela ação da pirimidina nucleosídeo difosfato quinase, ocorre a formação da lamivudina trifosfato (3TC-3P) (Moore et al., 1999). O anabólito ativo é o composto trifosforilado (3TC3P), sendo que o difosfato é o anabólito predominante e limitante da formação da 3TC-3P (Johnson et al., 1999).

Kewn e colaboradores (1997) investigaram o anabolismo intracelular da $3 \mathrm{TC}$ para dar origem à forma trifosfatada. Eles avaliaram dois tipos de células sangüíneas: mononucleares periféricas e monocíticas.

A 3TC-3P se liga ao DNA do HIV competindo com a 2'-desoxicitidina 5'-trifosfato. Devido à ausência do grupo hidroxila na posição 3' a cadeia de DNA não é alongada e não há replicação do HIV (Perry, Faulds, 1997).

A ZDV e a 3TC apresentam mecanismo comum de inibição da replicação do HIV. Ambas atuam inibindo a enzima transcriptase reversa viral, pois se ligam ao DNA pró-viral de linfócitos, ocasionando a terminação da cadeia de aminoácidos. Para se transformarem na forma ativa trifosfatada ambas são fosforiladas em reações intracelulares (Aoki, 1999). Para que esta associação medicamentosa seja eficaz, é necessário que o mecanismo de fosforilação de ambos seja enzimaticamente diferente. A ZDV é fosforilada pela timidina quinase, que não utiliza a 3TC como substrato (Veal et al., 1996).

Após fosforilação ambos os fármacos formam os anabólitos ativos 5 '-trifosfato que atuam inibindo a transcriptase reversa e terminam a extensão da cadeia de DNA pró-viral (Moore, Shaw et al., 1999). Os metabólitos fosforilados não são usualmente detectados no sangue (Moyer et al., 1999).

\section{RESISTÊNCIA AOS FÁRMACOS}

Estudos comprovam que a monoterapia com ZDV para pacientes portadores do HIV apresenta resistência em $50 \%$ dos indivíduos em seis meses e em quase todos após dois anos (Aoki, 1999).

A terapia com 3TC promove rápida seleção de mutação no códon 184 do gene do HIV. Esta é a região de codificação da enzima transcriptase reversa. Isto diminui parcialmente a sensibilidade ao fármaco. Porém, a carga viral permanece abaixo da linha de base em alguns pacientes. Esta mutação também reduz a sensibilidade do vírus à didanosina e à zalcitabina (Perry, Faulds, 1997).

Este mecanismo de resistência pode ser mediado por mutações nos códons 135 ou 333 do gene da transcriptase reversa. A resistência viral à ZDV e à $3 \mathrm{TC}$ combinadas foi observada apenas in vitro e em um pequeno número de casos clínicos isolados (Perry, Faulds, 1997).

\section{EFEITOS ADVERSOS}

As concentrações de ZDV necessárias ao tratamento anti-HIV são tóxicas para as células mielóides e eritóides progenitoras, o que explica a ocorrência de anemia e granulocitopenia durante a terapia. A incorporação da ZDV ao DNA mitocondrial pode explicar a miopatia observada em alguns pacientes (Aoki, 1999).

Os efeitos adversos da ZDV são mais comuns em portadores do HIV, com doença em estágio avançado. Em pacientes assintomáticos ou quase assintomáticos, especialmente aqueles submetidos a doses diárias reduzidas, os efeitos adversos são pouco comuns. O principal efeito adverso relatado pelo uso de ZDV é a mielossupressão (Aoki, 1999).

Anemia macrocítica e neutropenia ocorrem em 4-6 semanas após o início do tratamento dos pacientes com infecção assintomática e avançada. São observadas incidências de $2 \%$ a $37 \%$ com dose de $600 \mathrm{mg} /$ dia e $6 \%$ a $50 \%$

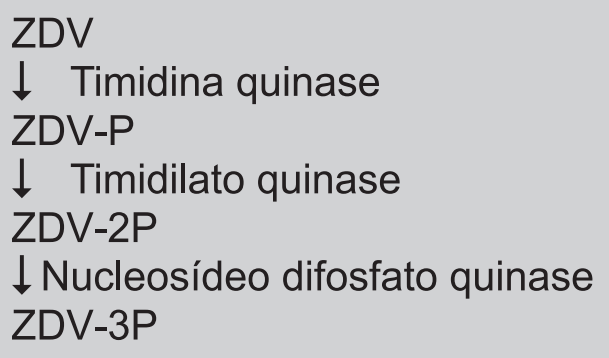

FIGURA 3 - Esquema do caminho de fosforilação intracelular da ZDV e 3TC (Kewn et al., 1997).
3TC

$\downarrow$ Desoxicitidina quinase 3TC-P $\downarrow$ Citidina monofosfato quinase 3TC-2P $\downarrow$ Nucleosídeo difosfato quinase 3TC-3P 
com dose de $1200 \mathrm{mg} /$ dia. Trombocitopenia foi observada em $12 \%$ dos pacientes tratados com ZDV. Estes efeitos mostraram-se reversíveis com a interrupção da terapia (Aoki, 1999).

Podem ser observados com relativa freqüência alguns efeitos menos graves como cefaléia intensa, mialgia, náusea e insônia. Outros efeitos raros, porém importantes, incluem miopatia, hepatomegalia com esteatose e acidose lática, além de anafilaxia (Aoki, 1999).

Moore e colaboradores (1999) realizaram estudos farmacocinéticos da 3TC. Eles avaliaram a administração em pacientes infectados por HIV, em doses de 150 e 300 $\mathrm{mg}$, duas vezes ao dia. Dentre os resultados, os estudos relataram que a $3 \mathrm{TC}$ é bem tolerada pelos pacientes. Os efeitos adversos mais descritos são distúrbios gastrointestinais. Efeitos indesejáveis como dores de cabeça, dores abdominais, náusea e fadiga podem ocorrer, porém com freqüência e intensidade bem inferiores aos observados com o uso conjunto da ZDV (Perry, Faulds, 1997).

Em pacientes com infecção por HIV em estágio avançado (contagem de CD4+ menor que 100 células/ $\mu \mathrm{L}$ ), o uso de 3TC pode não ser bem tolerado. Durante um estudo aplicando a terapia anti-HIV constituída pela $3 \mathrm{TC}$ em 89 crianças, foram relatados $8 \%$ de casos de pancreatites. Porém, este fato não foi comprovadamente atribuído a este fármaco (Perry, Faulds, 1997).

Em geral, a 3TC apresenta baixa toxicidade, que aumenta proporcionalmente ao acréscimo da dose. Ela é considerada bastante segura nas doses usuais que variam de 0,5 a $20 \mathrm{mg} / \mathrm{kg} / \mathrm{dia}$ (Zhou, Sommadossi, 1997). É improvável a indução pela 3TC de efeitos adversos hematológicos e hepáticos, clinicamente importantes, como neuropatias e miopatias (Johnson et al., 1999).

Foram realizados estudos de fase IV com 251 pacientes portadores de HIV-1, utilizando $150 \mathrm{mg}$ de $3 \mathrm{TC}$ duas vezes ao dia e $200 \mathrm{mg}$ de ZDV de 8 em 8 horas. Durante os testes foram relatados os seguintes efeitos adversos com sua freqüência: dor de cabeça (35\%), náusea $(33 \%)$ e mal-estar/fadiga $(27 \%)$. Não foram observados efeitos mais graves que os observados com a ZDV isoladamente (Johnson et al., 1999).

\section{FARMACOCINÉTICA}

Estudos da farmacocinética da ZDV mostraram que os dados obtidos tanto com voluntários sadios quanto com portadores do HIV são semelhantes. Apenas problemas renais graves, promovem pequena alteração dos parâmetros farmacocinéticos (Veal, Back, 1995).

Blum e colaboradores (1988) realizaram estudos de biodisponibilidade, utilizando doses múltiplas de ZDV. A pesquisa da qual participaram pacientes portadores do HIV comparou a administração de doses crescentes de ZDV por via intravenosa e oral com intervalos de administração de 4 e 8 horas. Em doses entre 2 e 15 mg/kg a ZDV apresenta farmacocinética linear. Doses elevadas podem ocasionar saturação das enzimas metabolizadoras e diminuição do CL/F (Acosta et al., 1996).

Um modelo de farmacocinética linear pode ser aplicado à administração da $3 \mathrm{TC}$ em doses múltiplas (Johnson et al., 1999), por via oral ou intravenosa. Johnson e colaboradores (1999) realizaram estudos em voluntários sadios para avaliar a farmacocinética da 3TC após administração oral de $300 \mathrm{mg}$ do fármaco. Os resultados obtidos de nove indivíduos foram os seguintes: concentração plasmática máxima $\left(\mathrm{C}_{\max }\right) 3798(3038-4747) \mu \mathrm{g} / \mathrm{L}$; tempo necessário para atingir $\mathrm{C}_{\text {max }}\left(\mathrm{t}_{\max }\right) 1,0(0,5-1,6) \mathrm{h}$; área sob a curva de concentração plasmática versus tempo (ASC) 15579 (13412-18096) $\mu \mathrm{g} . \mathrm{h} / \mathrm{L}$; meia-vida de eliminação $\left(\mathrm{t}_{1 / 2}\right) \beta 11,7 \mathrm{~h}$ e clearance oral aparente (CL/F) 19,3 L/h (Johnson et al., 1999).

Os parâmetros farmacocinéticos da $3 \mathrm{TC}$ obtidos de pacientes portadores de HIV-1 assintomáticos, HBV e voluntários sadios são os mesmos (Johnson et al., 1999). Em pacientes com grave ou moderado comprometimento renal, a ASC pode aumentar 270 a $500 \%$ em relação a voluntários sadios (Perry, Faulds, 1997).

Estudos mostraram que ocorreram alterações dos dados farmacocinéticos da 3TC e da ZDV, quando administradas isoladamente e em conjunto. A associação ocasionou aumento de $39 \%$ de $\mathrm{C}_{\max }$ da ZDV, o que não foi considerado clinicamente relevante (Johnson et al., 1999).

Moore, Shaw e colaboradores (1999) compararam a bioequivalência de comprimidos contendo 3TC e ZDV em associação e isoladamente (Tabela I). Foram avaliados também os efeitos da alimentação na absorção dos fármacos em ambos os casos. O estudo foi cruzado, realizado em 24 voluntários sadios de ambos os sexos. Eles foram divididos em três grupos. O primeiro recebeu um comprimido contendo $150 \mathrm{mg}$ de 3 TC e $300 \mathrm{mg}$ de ZDV em jejum. O segundo recebeu a mesma dose dos fármacos administrados em comprimidos separados em jejum. O terceiro grupo recebeu a associação cinco minutos após um café da manhã com alto teor de gordura. Os testes demonstraram a bioequivalência dos tratamentos e que embora após a alimentação o $t_{\max }$ tenha aumentado e o $\mathrm{C}_{\max }$ diminuído, a absorção da formulação não é alterada pela alimentação.

Os valores médios de área sob a curva (ASC) da concentração plasmática de lamivudina em função do tempo foram similares em grupos de pacientes que receberam o fármaco juntamente com alimento e com estômago vazio (Perry, Faulds, 1997). 
Atualmente, existem poucos estudos sobre os parâmetros farmacocinéticos das formas fosforiladas da ZDV e da 3TC. Devido a isto os resultados ainda não são conclusivos. Estudos realizados em portadores do HIV quantificaram a ZDV-3P e a $3 \mathrm{TC}-3 \mathrm{P}$ em células mononucleares periféricas sangüíneas. $\mathrm{O}$ método consistiu em extração em fase sólida dos compostos fosforilados utilizando soluções de cloreto de potássio. A quantificação foi efetuada por radioimunoensaio. Os resultados indicam que não há relação entre a concentração da ZDV plasmática e ZDV-3P intracelular. Embora tenha sido observada uma relação linear crescente entre o clearance oral peso-aparente ajustado da 3TC no plasma e a concentração intracelular da 3TC-3P esses resultados não foram estatísticamente significativos (Fletcher et al., 2000).

\section{ABSORÇÃO}

A ZDV é rápida e quase completamente absorvida por difusão passiva. $O$ processo segue cinética de primeira ordem e varia de 5 a mais de 40 minutos. A velocidade e a extensão da absorção são afetadas pelo conteúdo estomacal. Foram também observadas diferenças na velocidade de absorção e no tmax entre voluntários sadios e pacientes (Acosta et al., 1996).

A 3TC é altamente solúvel e permeável com rápida velocidade de dissolução. Conseqüentemente as doses orais são rapidamente absorvidas por difusão passiva através da parede intestinal. A absorção do fármaco não é afetada pela presença de alimentos (Johnson et al., 1999).

A absorção e a biodisponibilidade de agentes antiretrovirais em crianças portadoras do HIV podem ser afetadas significativamente pelos processos de crescimento e desenvolvimento pediátrico. A biodisponibilidade de ZDV em recém-nascidos pode variar devido à diminuição da glicuronidação hepática comparativamente a adultos (King et al., 2002).

Para se tornarem ativas a 3TC e a ZDV passam por processo de fosforilação nas células mononucleares periféricas sangüíneas. A formação intracelular dos compostos trifosforilados algumas vezes é dependente de ativação celular, como ocorre com a ZDV. A 3TC não necessita de ativação do processo de fosforilação intracelular.

Realizaram-se estudos avaliando a administração de doses de $3 \mathrm{TC}$ de 150 e $300 \mathrm{mg}$ duas vezes ao dia em portadores do HIV. Estes estudos concluíram que a dose de $150 \mathrm{mg}$ duas vezes ao dia produz máxima concentração do anabólito trifosforilado (3TC-3P). A elevação da dose não ocasiona significativo aumento da $3 \mathrm{TC}-3 \mathrm{P}$. Isto pode ser atribuído ao fato de a fosforilação ser processo saturável (Moore et al., 1999).

O estudo realizado por Moore e colaboradores (1999) avaliou parâmetros farmacocinéticos da fosforilação da 3TC. Os resultados mostraram que não há relação linear entre a farmacocinética da $3 \mathrm{TC}$ no soro e dentro das células.

A biodisponibilidade oral da ZDV é de aproximadamente $66 \%$, podendo ocorrer grande variabilidade individual (Balint, 2001).

Embora a $3 \mathrm{TC}$ apresente alta absorção e boa biodisponibilidade sistêmica, foram observadas alterações interpacientes. A biodisponibilidade variou de 53 a 105\% com uma dose de $100 \mathrm{mg}$. A biodisponibilidade absoluta da 3 TC é superior a $80 \%$ em adultos e cerca de $60 \%$ em crianças (Perry, Faulds, 1997).

TABELA I - Resumo das propriedades farmacocinéticas após administração de $150 \mathrm{mg}$ de 3TC e $300 \mathrm{mg}$ de ZDV em dois comprimidos separados e associados na presença e ausência de alimentos

\begin{tabular}{ccccc}
\hline Parâmetros & Condições & $\begin{array}{c}\text { Associação de fármacos } \\
\text { sem alimento }\end{array}$ & $\begin{array}{c}\text { Fármacos separados } \\
\text { sem alimento }\end{array}$ & $\begin{array}{c}\text { Associação de fármacos } \\
\text { com alimento }\end{array}$ \\
\hline $\mathrm{ASC}$ & $\mathrm{ZDV}$ & $2398,2 \pm 705,8$ & $2390,9 \pm 553,0$ & $2147 \pm 664,6$ \\
$(\mathrm{ng} . \mathrm{h} / \mathrm{mL})$ & $3 \mathrm{TC}$ & $6137,6 \pm 1234,0$ & $6374,2 \pm 1607,4$ & $6035,4 \pm 1160,6$ \\
$\mathrm{C}_{\max }$ & $\mathrm{ZDV}$ & $2008,3 \pm 809,9$ & $1992,6 \pm 636,1$ & $1139,2 \pm 587,8$ \\
$(\mathrm{ng} / \mathrm{mL})$ & $3 \mathrm{TC}$ & $1620,3 \pm 519,6$ & $1742,2 \pm 616,3$ & $1367,6 \pm 403,9$ \\
$\mathrm{t}_{\max }$ & $\mathrm{ZDV}$ & $0,57 \pm 0,45$ & $0,58 \pm 0,34$ & $1,07 \pm 0,66$ \\
$(\mathrm{~h})$ & $3 \mathrm{TC}$ & $0,91 \pm 0,48$ & $0,91 \pm 0,37$ & $1,86 \pm 0,94$ \\
$\mathrm{t}_{1 / 2}$ & $\mathrm{ZDV}$ & $1,50 \pm 0,24$ & $1,45 \pm 0,24$ & $1,53 \pm 0,41$ \\
$(\mathrm{~h})$ & $3 \mathrm{TC}$ & $9,98 \pm 2,78$ & $9,79 \pm 2,42$ & $10,52 \pm 5,32$ \\
$\mathrm{Cl} / \mathrm{F}$ & ZDV & 130 & 129 & 146 \\
$(\mathrm{~L} / \mathrm{h})$ & 3TC & 25,0 & 24,3 & 25,3 \\
\hline
\end{tabular}

* Os valores apresentados correspondem a média de 24 determinações \pm desvio padrão. 


\section{DISTRIBUIÇÃO}

Após absorção cerca de $25 \%$ da ZDV se liga a proteínas plasmáticas. Sua passagem para as células ocorre por difusão passiva, devido a sua relativa lipossolubilidade. Ela é amplamente distribuída pelo organismo, chegando até o sêmen, fluido cérebro espinhal, e leite materno. Estudos realizados em 6 mulheres determinaram que a razão entre a quantidade de ZDV no leite e no soro é de 1,48 (Acosta et al., 1996).

Estudos sobre a relação entre peso corpóreo e a dose de ZDV ainda não são conclusivos. Alguns autores avaliaram a sua farmacocinética pelo método não-compartimental e concluíram que não é necessário individualizar a dose. Dados recentes sugerem relação significativa entre o peso corpóreo e o clearance da ZDV (Acosta et al., 1996). A dose da terapêutica anti-retroviral em crianças deve ser individualiza baseando-se na área da superfície corporal, que é calculada por meio do peso e da altura dos pacientes (King et al., 2002).

Pesquisas farmacocinéticas utilizando modelo não compartimental determinaram que o volume aparente de distribuição (Vd/F) é 121,2L (Zhou, Sommadossi, 1997), sendo o volume médio de distribuição $1,3 \mathrm{~L} / \mathrm{kg}$ e a porcentagem de fármaco que se liga a proteínas plasmáticas menor que 36\% (Perry, Faulds, 1997).

A 3 TC é amplamente distribuída e penetra livremente nos tecidos a partir da circulação sistêmica. Ela se difunde livremente através da placenta da circulação materna para a circulação fetal. A razão entre a concentração da 3TC no plasma e no leite materno é de 1:1 (Johnson et al., 1999).

\section{BIOTRANSFROMAÇÃO}

A ZDV sofre eliminação pré-sistêmica hepática. O principal caminho metabólico da ZDV é a glicuronidação (GZDV). Cerca de 60 a 70\% do fármaco são eliminados pela urina sob a forma inativa (GZDV) e aproximadamente $1 \%$ é eliminado sob a forma ativa (ZDV-TP). O terceiro passo metabólico é ocasionado por uma reação de redução com formação de 3'-amino-3'-desoxitimidina, que possui potencial citotoxicidade (Veal, Back, 1995). Alguns fármacos como a probenecida, o naproxeno e o fluconazol, que às vezes são co-administrados em pacientes portadores do HIV, podem interferir na glicuronidação e ocasionar aumento da atividade da ZDV, bem como da sua toxicidade (Veal, Back, 1995).

Pequena porção da 3TC administrada é biotransformada antes de ser eliminada. Aproximadamente 5 a $10 \%$ do fármaco são biotransformados ao metabólito ina- tivo trans-sulfóxido, que é excretado na urina até no máximo 12 horas após a dose oral (Johnson et al., 1999).

\section{ELIMINAÇÃO}

A eliminação plasmática da ZDV é rápida, com meia-vida de eliminação de 1 a 1,5 h. Porém, a depleção dos nucleotídeos intracelulares da ZDV é mais lenta, o que permite, durante o tratamento, um intervalo de doses de oito horas (Aoki, 1999).

A 3TC apresenta meia-vida de eliminação $\left(\mathrm{t}^{1} / 2\right) \beta$ da ordem de 8,5 a 11,5 horas, sendo que $70 \%$ do fármaco são excretados na forma inalterada na urina até 24 horas após a dose. Sua eliminação renal ocorre por filtração e secreção tubular ativa (Johnson et al., 1999). A meia-vida de eliminação intracelular dos anabólitos fosforilados é de 10,5 a 15,5 h (Perry, Faulds, 1997).

\section{INTERAÇÕES MEDICAMENTOSAS}

O fluconazol ocasiona decréscimo de $43 \%$ no clearance oral aparente da ZDV. As mudanças farmacocinéticas indicam que $400 \mathrm{mg}$ de fluconazol inibem a principal via metabólica da ZDV, ou seja, sua biotransformação em compostos glicuronados (GZDV) (Veal, Back, 1995).

A 3 TC tem pequeno potencial para interagir com outros fármacos, quando administrados conjuntamente. Modelos in vitro de absorção mostraram que não há diferenças significativas entre a absorção da $3 \mathrm{TC}$, quando administrada conjuntamente com zidovudina, zalcitabina, didanosina, aciclovir, probenecida, trimetoprima, sulfametoxazol, ranitidina ou cimetidina. Fármacos excretados por via renal, como a trimetoprima e o sulfametoxazol, podem inibir competitivamente a eliminação da 3TC, aumentando a área sob a curva de concentração plasmática do fármaco versus tempo (ASC) e diminuindo o clearance renal aparente (CL/F) (Perry, Faulds, 1997). O clotrimoxazol ao ser administrado conjuntamente com 3TC ocasiona aumento da ASC de $44 \%$ e do CL/ F de 29,0\% (Johnson et al., 1999).

A 3TC e a zalcitabina são ativadas por mecanismo de fosforilação, que utiliza as mesmas enzimas. Devido a tal fato, a zalcitabina tem sua atividade bastante prejudicada na presença de 3TC (Perry, Faulds, 1997).

\section{TRANSMISSÃO VERTICAL}

Vários estudos têm sido efetuados com o objetivo de evitar a transmissão do vírus HIV de mãe para filho duran- 
te a gestação e o parto (Mandelbrot et al., 2001). A eficácia da terapia anti-retroviral em prevenir a transmissão vertical do HIV foi demonstrada primeiramente em 1994 (Connor et al., 1994).

Estudos foram realizados com administrações orais da ZDV entre a $14^{\mathrm{a}}$ e a $34^{\mathrm{a}}$ semanas de gestação em mulheres grávidas HIV-positivas. Durante o parto, a ZDV foi administrada via endovenosa e posteriormente ocorreu administração oral ao recém-nascido. Estes procedimentos reduziram a freqüência de infecção neonatal pelo HIV (Aoki, 1999). A ZDV atravessa a barreira placentária por difusão passiva, sendo que $70 \%$ do fármaco se difundem livremente nos dois sentidos da placenta. Estudos indicam que concentrações de 1 a $2 \%$ do fármaco na circulação materna atingem a concentração efetiva no feto (Acosta et al., 1996).

Moodley e colaboradores (1998) avaliaram a farmacocinética da 3 TC em 20 mulheres grávidas infectadas pelo HIV. Todas as pacientes estavam na $38^{\circ}$ semana de gestação. Dez gestantes receberam, por via oral, $300 \mathrm{mg}$ de $3 \mathrm{TC}$ a cada 12 horas e as outras dez foram medicadas com $150 \mathrm{mg}$ de $3 \mathrm{TC}$ e $300 \mathrm{mg}$ de ZDV a cada 12 horas. Foram determinados alguns parâmetros farmacocinéticos, como: concentração plasmática máxima (Cmax) e o tempo necessário para atingir esta concentração (tmax), ASC, meia-vida de eliminação ( $\left.\mathrm{t}^{1} / 2\right) \beta \mathrm{e} \mathrm{CL} /$ $\mathrm{F}$, cujos valores foram semelhantes aos obtidos em mulheres adultas não grávidas (Tabela II).

\section{CONCLUSÃO}

Atualmente a terapêutica anti-retroviral é composta por associações de fármacos. O uso de vários medicamentos em diferentes horas do dia diminui a adesão pelos portadores do HIV ao tratamento. A associação de lamivudina e zidovudina em um mesmo medicamento é uma tentativa de diminuir os erros e o abandono durante o tratamento.

A associação não acarreta aumento de efeitos tóxicos e pode ocasionar diminuição da resistência aos fármacos. Estes são indicados, também, para a prevenção na transmissão vertical do HIV. Não são significativas as alterações nos parâmetros farmacocinéticos dos fármacos isoladamente ou contidos na mesma formulação, embora sejam observadas diferenças em parâmetros obtidos de voluntários sadios e portadores do HIV.

\section{ABSTRACT}

Antiretroviral activity and pharmacokinetics properties of lamivudine and zidovudine association

Lamivudine (3TC) and zidovudine (ZDV) are antiretroviral drugs used in the treatment of HIV infection. They are nucleoside analogues that inhibit HIV-1 reverse transcriptase. These drugs are anabolized intracellularly by a stepwise process and form an active triphosphate

TABELA II - Parâmetros farmacocinéticos da 3TC em monoterapia e associada a ZDV obtidos após uma dose em pacientes grávidas antes do parto e no estado de equilíbrio (steady-state) após o parto (Moodley et al., 1998)

\begin{tabular}{|c|c|c|c|}
\hline $\begin{array}{l}\text { Parâmetros } \\
\text { farmacocinéticos }\end{array}$ & $\begin{array}{l}\text { 3TC em monoterapia } \\
\text { (dose: } 300 \mathrm{mg} \\
2 \text { vezes ao dia) }\end{array}$ & $\begin{array}{c}3 \mathrm{TC}+\mathrm{ZDV} \\
\text { (dose: } 150 \mathrm{mg} \\
2 \text { vezes ao dia) }\end{array}$ & Comparação $^{a}$ \\
\hline \multicolumn{4}{|l|}{ Primeira dose } \\
\hline $\mathrm{ASC}_{0-\not}(\mathrm{ng} \cdot \mathrm{h} / \mathrm{mL})^{\mathrm{b}}$ & $10047(8482-11921)$ & 4933 (3997-6089) & $1,02(0,83-1,25)$ \\
\hline $\mathrm{C}_{\text {max }}(\mathrm{ng} / \mathrm{mL})^{\mathrm{b}}$ & 2504 (1996-3140) & $1313(1070-1610)$ & $0,95(0,75-1,2)$ \\
\hline $\mathrm{t}_{\max }(\mathrm{h})$ & $1,5(0,5-2,0)$ & $1,0(0,5-2,0)$ & - \\
\hline $\mathrm{CL} / \mathrm{F}(\mathrm{L} / \mathrm{h} / \mathrm{Kg})$ & $0,41(0,34-0,49)$ & $0,44(0,35-0,55)$ & $0,94(0,75-1,17)$ \\
\hline $\mathrm{T}_{1 / 2}(\mathrm{~h})$ & $2,3^{\mathrm{b}}(2,0-2,6)$ & $2,3^{\mathrm{b}}(2,0-2,7)$ & $0,99(0,85-1,14)$ \\
\hline \multicolumn{4}{|c|}{ Uma semana pós-parto } \\
\hline $\mathrm{ASC}_{\mathrm{t}}(\mathrm{ng} \cdot \mathrm{h} / \mathrm{mL})^{\mathrm{b}}$ & $9896(7832-12505)$ & $5082(3749-6889)$ & $0,97(0,73-1,31)$ \\
\hline $\mathrm{C}_{\max }(\mathrm{ng} / \mathrm{mL})^{\mathrm{b}}$ & $2433(1761-3363)$ & $1210(981-1492)$ & $1,01(0,75-1,35)$ \\
\hline$t_{\text {max }}(h)$ & $1,0(0,5-4,0)$ & $1,5(0,5-4,0)$ & - \\
\hline $\mathrm{CL} / \mathrm{F}(\mathrm{L} / \mathrm{h} / \mathrm{Kg})$ & $0,45(0,35-0,58)$ & $0,46(0,32-0,66)$ & $0,98(0,70-1,37)$ \\
\hline $\mathrm{T}_{1 / 2}(\mathrm{~h})$ & $5,9(4,1-8,7)$ & $6,6(4,9-9,2)$ & $0,90(0,62-1,32)$ \\
\hline
\end{tabular}

${ }^{a}$ Média geométrica usando o método dos quadrados mínimos (intervalo de confiança de 90\%)

${ }^{\mathrm{b}} \mathrm{AUC}$ e $\mathrm{C}_{\max }$ dose-normalizados por comparações 
anabolite, which is used by HIV-1 reverse transcriptase and effectively terminates chain extension. The use of monotherapy is associated with a delay in the emergence of resistant mutants. The combination therapy would therefore seem to be potentially useful for rapid reduction in virus load. Adverse events were similar in both regimen, with the exception of lower incidence of anemia when used only 3TC. Both drugs are rapidly absorbed by passive diffusion through the intestinal wall. They are distributed into total body fluid and have a good bioavailability. They diffuse freely across the placenta from the maternal circulation to the fetal circulation. The glucuronidation is the main pathway of ZDV metabolism. About 60 to $70 \%$ of the drug undergoes biotransformation. The ZDV has a short elimination half-life from 1 to 1.5 hour. The $3 T C$ is primarily eliminated unchanged through by renal excretion. A pharmacologically inactive trans-sulphoxide metabolite has been determined and corresponds to $5 \%$ to $10 \%$ of $3 T C$ dose. The terminal elimination half-life of $3 T C$ was from 8.5 to 11.5 hours.

UNITERMS: Lamivudine. Zidovudine. Antiretroviral drugs. Pharmacokinetics

\section{REFERÊNCIAS BIBLIOGRÁFICAS}

ACOSTA, E. P.; PAGE, L. M.; FLETCHER, C. V. Clinical pharmacokinetics of zidovudine. Clin. Pharm., v. 30, n. 4, p. 251-262, 1996.

AMATO NETO, V.; MEDEIROS, E. A. S.; KALLAS, E. G.; LEVI, G. C.; BALDY, J. L. S.; MEDEIROS, R. S. S. AIDS na prática médica. São Paulo: Sanvier, 1996. 153p.

AOKI, F. Y. Infecções virais. In: PAGE, C. P.; CURTIS, M. J.; SUTTER, M. C.; WALKER, M. J. A.; HOFFMAN, B. B. Farmacologia Integrada. São Paulo: Manole, 1999. p. 445-460.

AYMARD, G.; LEGRAND, M.; TRICHEREAU, N.; DIQUET, B. Determination of twelve antiretroviral agents in human plasma sample using reversed-phase high-performance liquid chromatography. $J$. Chromatogr. B, v.744, p. 227-240, 2000.

BALINT, G. A. Antiretroviral therapeutic possibilities for human immunodeficiency virus/acquired imunodeficiency syndrome. Pharmacol. Ther., v 89, p. 17-27, 2001.
BARRY, M.; MULCAHY, F.; MERRY, C.; GIBBONS, S.; BACK, D. Pharmacokinetics and potential interactions amongst antiretroviral agents used to treat patients with HIV infection. Clin. Pharmacokinet., v. 36, n. 4, p. 289304, 1999.

BLUM, R.; LIAO, H. T. S.; GOOD, S. S.; MIRANDA, P. Pharmacokinetics and biovailability of zidovudine in humans. Am. J. Med., v.85, S. 2A, p. 189-194, 1988.

BRASIL. Ministério da Saúde. Boletim epidemiológico editorial. Disponível em <http:// www.aids.gov.br/ sitebol/inferior.htm>. Acesso em: 01 de julho de 2002a.

BRASIL. Ministério da Saúde. Documentos, recomendações e relatórios técnicos. Disponível em $<\mathrm{http}: / /$ www.aids.gov.br/documentos.htm>. Acesso em: 01 de julho de $2002 b$.

COHN, J. A. Recent advances HIV-1 infection $-\mathrm{I}$. BMJ., v. 314, p. 487-491, 1997.

CONNOR, E. M.; SPERLING, R.; GELBER, R.; KISELEV, P.; SCOTT, G.;O’SULLIVAN, M. J.; VANDYKE, R.; BEY, M.; SHEARER, W.; JACOBSON, R. L.; JIMENEZ, E.; O'NEILL, E.; BAZIN, B.; DELFRAISSY, J. F.; CULNANE, M.; COOMBS, R.; ELKINS, M.; MOYE, J.; STRATTON, P.; BALSLEY, J. Reduction of maternal-infant transmission of human immunodeficiency virus type 1 with zidovudine treatement. N. Engl. J. Med., v. 331, p. 1173-1180, 1994.

ERON, J. J.; YETZER, E. S.; RUANE, P. J.; BECKER, S.; SAWYERR, G. A.; FISHER, R. L.; TOLSON, J. M.; SHAEFER, M. S. Efficacy, safety and adherence with a twice-daily combination lamivudine/zidovudine tablet formulation, plus a protease inhibitor, in HIV infection. AIDS., v. 14, n. 6, p. 671-681, 2000.

FERNADES, C. Estudo de equivalência farmacêutica de comprimidos de lamivudina $150 \mathrm{mg}$. Belo Horizonte, 2001. 189 p. (Dissertaçãode Mestrado. Faculdade de Farmácia, Universidade Federal de Minas Gerais).

FLETCHER, C. V.; RAWLE, S. P.; KAKUDA, T. N.; ANDERSON, P. L.; WELLER, D.; LANE, R. B.; BRUNDAGE, R. C.; REMMEL, R. P. Zidovudine triphosphate and lamivudine triphosphate concentrationresponse relationships in HIV-infected persons. AIDS, v.14, n. 14, p. 2137-2144, 2000. 
FRIJUS-PLESSEN, N.; MICHAELIS, H.C.; FOTH, H.; KAHL, G.F. Determination of 3'-azido-3'deoxythymidine, 2',3'-dideoxycytidine, 3'-fluoro-3'deoxythymidine and 2',3'-dideoxyinosine in biological samples by high-performance liquid chromatography. $J$. Chromatogr. Biom. Appl., v. 534, p. 101-107, 1990.

GLAXO - editorial. Disponível em $<$ http:// www.glaxo.com.br >. Acesso em: 01 de julho de 2002.

GULICK, R. M.; MELLORS, J. W.; HAVLIR, D.; ERON, J. J.; GONZALEZ, C.; McMAHON, D.; RICHMAN, D. D.; VALENTINE, F. T.; JONAS, L.; MEIBOHM, A.; EMINI, E. A.; CHODAKEWITZ, J. A. Treatment with indinavir, zidovudine and lamivudine in adults with human immunodeficiency virus infection and prior antiretroviral therapy. N. Engl. J. Med., v. 337, n. 11, p. 734- 739, 1997.

HAVLIR, D. V.; LANGE, J. M. A. New antivirals and new combintions. AIDS, v.12, Sup. A, p. S165-S174, 1998.

HARKER, A. J.; EVANS, G. L.; HAWLEY, A. E.; MORRIS, D. M. High-performance liquid chromatographic assay for 2'-deoxy-3'-thiacutidine in human serum. J. Chromatogr. B: Biom. Appl., v. 657, p. 227-232, 1994.

HENRY, K.; ERICE, A.; TIERNEY, C.; BALFOUR JR, H. H.; FISCHL, M. A.; KMACK, A.; LIOU, S. H.; KENTON, A.; HIRSCH, M. S.; PHAIR, J.; MARTINEZ, A.; KAHN, J. O. A randomized, controlled, double-blind study comparing the survival benefit of four different reverse transcriptase inhibitor therapies (three-drug, two-drug, and alternating drug) for the treatment of advanced AIDS. J. Acquir. Imm. Defic. Synd. and human retrovirol., v. 19, n. 4, p. 339-349, 1998.

HOETELMANS, R. M. W.; PROFIJT, M.; MEENHORST, P. L.; MULDER, J. W.; BEIJNEN, J. H. Quantitative determination of (-)-2'-deoxy-3'-thiacytidine (lamivudine) in human plasma, saliva and cerebrospinal fluid by high-performance liquid chromatography with ultraviolet detection. J. Chromatogr. B., v.713, p. 387394, 1998.

HORNEFF, G.; ADANS, O.; WAHN, V. Pilot study of zidovudine-lamivudine combination therapy in vertical HIV-infected antiretroviral-naive children. AIDS, v. 12, n. 5, p. 489-494, 1998.
JOHNSON, M. A.; MOORE, K. H. P.; YUEN, G. J.; BYE, A.; PAKES, G. E. Clinical pharmacokinetics of lamivudine. Clin. Pharmacokinet., v. 36, n. 1, p. 41-66, 1999.

JOZWIAKOWSKI, M. J.; NGUYEN, N. A. T.; SISCO, J. M.; SPANCAKE, C. W. Solubility behavior of lamivudine crystal forms in recrystallization solvents. $J$. Pharm. Sci., Washington, v.85, n.2, p.193-199, 1996.

KEWN, S.; VEAL, G. J.; HOGGARD, P. G.; BARRY, M. G.; BACK, D. J. Lamivudine (3TC) phosphorylation and drug interations in vitro. Biochem. Pharmacol., v. 54, p. 589-595, 1997

KING, R. J.; KIMBERLIN, D. W.; ALDROVANDI, G. M.; ACOSTA, E. P. Antiretroviral pharmacokinetics in the paediatric population. Clin. Pharmacokinet., v. 44, n.14, p. 1115-1133, 2002.

KYOKO, H.; ETSUKO, U.; MASUMITSU, T. Physicochemical properties and stability of Epivir tablets. Kagaku Ryoho no Ryoiki, Osaka, v.13, p. 1170-1172, 1997.

LEGRAND, M.; COMETS, E.;AYMARD, G.; TUBIANA, R.; KATLAMA, C.; DIQUET, B. An in vivo pharmacokinetic/pharmacodynamic model for antiretroviral combination. HIV Clin. Trials., v. 4, n.3, p.170-83, 2003.

MANDELBROT, L.; LANDREAU-MASCARO, A.; REKACEWICZ, C.; BERRBI, A., BÉNIFLA, J. L.; BURGARD, M.; LACHASSINE, E.; BARRET, B.; CHAIX, M. L.; BONGAIN, A., CIRARUVIGNERON, N.; CRENNHEBRT, C.; DELFRAISSY, J.F.; ROUZIOUX, C.; MAYAUX, M. J.; BLANCHE, S. Lamivudine-zidovudine combination for prevention of maternal-infant transmission of HIV-1. JAMA, v. 285, n. 16, p. 2083-2093, 2001.

MESPLET, N.; MORIN, P.; FRANÇOIS, C. AGROFOGLIO, L. A. Simultaneous quantification of nicleoside HIV-1 reverse transcriptase inhibitors by short-end injection capillary eletrochromatography on a $\beta$-cyclodextrinblonded silica stationary phase. J. Chromatogr. A., v. 927, p. 161-168, 2001. 
MOODLEY, J.; MOODLEY, D.; PILLAY, K.; COOVADIA, H.; SABA, J.; VAN LEEUWEN, R.; GOODWIN, C.; HARRIGAN, P. R.; MOORE, K. H.; STONE, C.; PLUMB, R.; JOHNSON, M. A. Pharmacokinetics and antiretroviral activity of lamivudine alone or when coadministered with zidovudine in HIV-infected pregnant women and their offspring. J. Infect. Dis., v. 178, p. 13271333, 1998.

MONTANER, J. S. G.; REISS, P.; COOPER, D.; VELLA, S.; HARRIS, M.; CONWAY, B.; WAINBERG, M. A.; SMITH, D.; ROBINSON, P.; HALL, D.; MYERS, M.; LANGE, J. M. A. A randomized, double-blind trial comparing combinations of nevirapine, didanosine and zidovudine for HIV-infected patients. JAMA, v. 279, n.12, p. 930-937, 1998.

MOORE, J. D.; VALETTE, G.; DARQUE, A.; ZHOU, X. J.; SOMMADOSSI, J. P. Simultaneous quantitation of the 5'- triphosphate metabolites of zidovudine, lamivudine, and stavudine in peripheral mononuclear blood cells of HIV infected patients by high-performance liquid chromatography tandem mass spectrometry. J. Am. Soc. Mass Spectrom., v. 11, p. 1134-1143, 2000.

MOORE, K. H. P; BARRETT, J. E.; SHAW, S.; PAKES, G. E.; CHURCHUS, R.; KAPPOR, A.; LLOYD, J.; BARRY, M. G; BACK, D. The pharmacokinetics of lamivudine phosphorilation in peripheral blood mononuclear cells from patients infected HIV-1. AIDS, v. 13, n. 16, p. 2239-2250, 1999.

MOORE, K. H. P.; SHAW, S.; LAURENT, A. L.; LLOYD, P.; DUNCAN, B.; MORRIS, D. M.; O’MARA, M. J.; PAKES, G. E. Lamivudine/zidovudine as a combined formulation tablet: bioequivalence compared with lamivudine and zidovudine administered concurrently and the effect of food on absorption. J. Clin. Pharmacol., v. 39, p. 593-605, 1999.

MOYER, T. P.; TEMESGEN, Z.; ENGER, R.; ESTES, L.; CHARLSON, J.; OLIVER, L.; WRIGHT, A. Drug monitoring of antiretroviral therapy for HIV-1 infection: method validation and results of a pilot study. Clin. Chem., v. 45, n. 9, p. 1465-1476, 1999.
PEREIRA, A. S.; KENNEY, K. B.; COHEN, M. S.; HALL, J. E.; ERON, J. J.; TIDWELL, R. R.; DUNN, J. A. Simultaneous determination of lamivudine and zidovudine concentrations in human seminal plasma using high-performance liquid chromatography and tandem mass spectrometry. J. Chromatogr. B., v. 742, p. 173-183, 2000.

PERRY, C. M.; FAULDS, D. Lamivudine. A review of its antiviral activity, pharmacokinetic properties and therapeutic efficacy in the management of HIV infection. Drugs, v.53, n.4, p. 657-680, 1997.

STYRT, B. A.; PIAZZA-HEPP,T. D.; CHIKAMI, G. K. Clinical toxicity of antiretroviral nucleoside analogs. Antiv. Res., v.31, p. 121-135, 1996.

SIMON, V. A.; THIAM, M. D.; LIPFORD, L. C. Determination of serum levels of thirteen human immunodeficiency virus-suppressing drugs by highperformance liquid chromatography. J. Chromatogr. A., v. 913, p. 447-453, 2001.

VEAL, G. J.; HOGGARD, P. G.; BARRY, M. G.; KHOO, S.; BACL, D. J. I nteration between lamivudine (3TC) and other nucleoside analogues for intracellular phosphorilation. AIDS., v. 10, n. 5, p. 546-548, 1996.

VEAL, G. J.; BACK, J. D. Metabolism of zidovudine. Gen. Pharmacol., v. 26, n. 7, p. 1469-1475, 1995.

ZHOU, X. L.; SOMMADOSSI, J. P. Rapid quantitation of()-2'-deoxy-3'-thiacytidine in human serum by highperformance liquid chromatography with ultraviolet detection. J. Chromatogr. B., v. 691, p. 417-424, 1997.

Recebido para publicação em 10 de fevereiro de 2003. 